\title{
Integrated Analysis Reveals That Long Non-Coding RNA TUBA4B Can Be Used as a Prognostic Biomarker in Various Cancers
}

\author{
Ting Zhang Dong-ming Wu Shi-hua Deng Rong Han Teng Liu Jing Li \\ Ying $\mathrm{Xu}$
}

Clinical Laboratory, The First Affiliated Hospital of Chengdu Medical College, Chengdu, China

\section{Key Words}

Tuba4b • LncRNA • Prognosis • Gene Expression Omnibus • Meta-analysis • FISH

\begin{abstract}
Background/Aims: Recent studies have reported the importance of tubulin alpha 4b (TUBA4B), a long non-coding RNA, in the development of several cancers; however, studies on its clinical significance are rare. In the present meta-analysis, we investigated whether TUBA4B can be used as a prognostic biomarker in human cancers. Methods: A comprehensive search was performed in PubMed, Embase, Web of Science, and the Gene Expression Omnibus databases. Hazard ratios from individual studies were calculated and pooled using a random-effects or fix-effects model. The pooled hazard ratio (HR) with $95 \%$ confidence interval $(\mathrm{CI})$ was used to evaluate the value of TUBA4B. The expression of TUBA4B was evaluated in lung cancer tissue arrays by fluorescence in situ hybridization assay. Additionally, a sensitivity analysis and Begg's test were conducted. Results: We found that TUBA4B was significantly correlated with overall survival (OS) (HR = 1.33, 95\% CI: 1.16-1.52, $P=0.000)$, disease-free survival (DFS; $H R=1.25$, 95\% CI: $1.06-1.48, P=0.007$ ), and recurrence-free survival (RFS; $\mathrm{HR}=1.42,95 \% \mathrm{CI}: 1.26-1.60$, $P=0.000)$. In addition, TUBA4B was a risk factor for lung cancer (HR $=1.24,95 \% \mathrm{CI}: 1.03-1.49$, $P=0.021)$, colon cancer ( $\mathrm{HR}=1.67,95 \% \mathrm{CI}: 1.02-2.74, P=0.042)$, breast cancer $(\mathrm{HR}=1.52,95 \%$ CI: $1.10-2.12, P=0.012)$, and ovarian cancer (HR $=1.67,95 \%$ CI: $1.18-2.36, P=0.004)$. Moreover, LnCRNA-TUBA4B was significantly lower expression in tumor tissues than normal lung tissues $(P<0.001)$. The expression of IncRNA-TUBA4B was decreased with the progression of lung cancer stage. A subgroup meta-analysis based on data resource, sample size, region, patient numbers, and tumor type was further performed. Our studies revealed that tumor tissues with low levels of TUBA4B was significantly associated with short OS, DFS, and RFS in cancer patients. Conclusion: The present findings suggest that TUBA4B can be a novel biomarker for the prognosis of various cancers.
\end{abstract}




\section{Cellular Physiology Cell Physiol Biochem 2018;49:530-544 \begin{tabular}{l|l|l} 
and Biochemistry Published online: 29 August, 2018 & $\begin{array}{l}\text { (c) 2018 The Author(s). Published by S. Karger AG, Basel } \\
\text { www.karger.com/cpb }\end{array}$
\end{tabular} \\ Zhang et al.: TUBA4B as a Prognostic Biomarker in Various Cancers}

\section{Introduction}

According to the GLOBOCAN estimates, approximately 14.1 million new cancer cases and 8.2 million deaths occurred worldwide in 2012 [1]. Cancer has become an increasingly serious problem worldwide. Early diagnosis and treatment are critical to improve the prognosis and survival of cancer patients. Furthermore, tumor molecular markers are of a great practical value in tumor screening, diagnosis, and prognosis; in evaluation of treatment efficacy; and for the follow-up of high-risk populations [2]. The molecular mechanisms underlying cancer development remain unclear; as such, the overall rate of cancer-related deaths are expected to increase. Therefore, identifying novel biomarker for diagnosis or prognosis is necessary to develop better therapeutic strategies to control cancer.

Recently, long non-coding RNAs (lncRNAs) have been identified to play important role as critical regulators of prognosis, pathogenesis, and biological processes in various cancers [3-5]. Furthermore, IncRNAs have important potential applications in the diagnosis and treatment of malignant tumors [6-8]. Recent studies have shown that various lncRNAs can act as tumor markers [9-11].

Tubulin alpha $4 \mathrm{~b}$ (TUBA4B) is a human protein significantly related with tumor progression. The low expression of TUBA4B can promote cell proliferation, advanced TNM stage, and lymph node metastasis in non-small-cell lung cancer [12]. Furthermore, TUBA4B is closely related with cell proliferation, migration, pathological grade, International Federation of Gynecology and Obstetrics stage, and lymph node metastases in ovarian cancer, and an increased expression of IncRNA-TUBA4B can attenuate the activation of ERK and AKT signaling pathways [13]. Thus, low levels of TUBA4B expression may be related to tumor prognosis.

However, most studies on TUBA4B are limited by their small sample size and discrete outcomes, and only few studies have investigated its prognostic significance in clinical tumor specimens. The Gene Expression Omnibus (GEO) database stores a massive amount of information on gene expression profiles in many tumor types. TUBA4B gene expression has been measured in this data set. Therefore, we systematically analyzed all previously published literature and the GEO database and performed a quantitative meta-analysis to evaluate the value of TUBA4B as a prognostic marker in human cancers.

\section{Materials and Methods}

\section{Study strategy}

Systematic literature searches were conducted in PubMed, Embase, and Web of Science. The literature search was conducted up to October 2017 and was limited to the English language. To increase the sensitivity of the search, both MeSH terms and free words were used. The search terms included "TUBA4B," "Tubulin, Alpha 4b," "long non-coding RNA" or "IncRNA," "cancer" or "carcinoma," "tumor" or "neoplasm," and "prognosis" or "survival." We also obtained microarray data, including overall survival (OS), disease-free survival (DFS), or recurrence-free survival (RFS), from the GPL570 platform (Affymetrix Human Genome U133 Plus 2.0 Array, HG-U133_Plus_2) from the GEO database.GEO database come from a variety of sources. To reduce data source variability, we selected only one platform (GPL570 platform) to minimize the impact on the heterogeneity in latter analysis.

\section{Study selection}

We manually searched and retrieved references for potentially missing literatures. First, the cited articles were excluded from duplicates, and then titles and abstracts were carefully scanned to eliminate irrelevant studies. The inclusion criteria are as follows: (a) TUBA4B expression in human tissues was measured and analyzed; (b) the relationship between TUBA4B expression and OS, DFS, or RFS was identified; (c) the survival curve or sufficient relevant data were provided to obtain hazard ratios (HR) for survival rates and their $95 \%$ confidence intervals (95\% CI). 


\section{Cellular Physiology Cell Physiol Biochem 2018:49:530-544

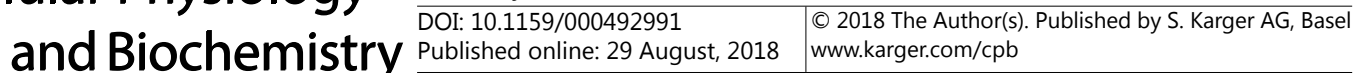 \\ Zhang et al.: TUBA4B as a Prognostic Biomarker in Various Cancers}

Table 1. Characteristics of articles included in the meta-analysis. *1 denoted as extracting HRs from KaplanMeier curve; 2 denoted as obtaining HRs directly from publications. OS: overall survival; UA: univariate analysis; MA: multivariate analysis

\begin{tabular}{|c|c|c|c|c|c|c|c|c|c|c|c|}
\hline Study & Year & Region & $\begin{array}{l}\text { Tumor } \\
\text { type }\end{array}$ & $\begin{array}{l}\text { Sample } \\
\text { size }\end{array}$ & $\begin{array}{l}\text { Clinical stage } \\
\text { of tumor }\end{array}$ & $\begin{array}{l}\text { Test methods of } \\
\text { TUBA4B expression }\end{array}$ & Elevated TUBA4B & $\begin{array}{l}\text { Outcome } \\
\text { measures }\end{array}$ & $\begin{array}{l}\text { Survival } \\
\text { analysis }\end{array}$ & Method* & $\begin{array}{l}\text { NOS } \\
\text { score }\end{array}$ \\
\hline $\begin{array}{l}\text { Chen et } \\
\text { al }\end{array}$ & 2016 & China & $\begin{array}{l}\text { Lung } \\
\text { cancer }\end{array}$ & 114 & $\begin{array}{l}\text { Stage I, II and } \\
\text { IIIA }\end{array}$ & qRT-PCR & $\begin{array}{ll}\text { Significantly } & \text { lower } \\
(\mathrm{P}<0.001) & \end{array}$ & os & No & 1 & $7 / 9$ \\
\hline $\begin{array}{l}\text { Zhu et } \\
\text { al }\end{array}$ & 2017 & China & $\begin{array}{l}\text { Ovarian } \\
\text { cancer }\end{array}$ & 116 & Stage I-IV & qRT-PCR & $\begin{array}{l}\text { Significantly lower } \\
(\mathrm{P}<0.05)\end{array}$ & OS & $\mathrm{UA}$ and MA & 2 & $8 / 9$ \\
\hline
\end{tabular}

The exclusion criteria are as follows: (a) studies were excluded if they were letters, case reports, reviews, or conference reports; (b) the required data could not be extracted or calculated from the original article; (c) the article could not be found in full or had been published repeatedly. When the same data subsets were published in more than one article, only the latest publication was included.

\section{Data extraction}

Eligible data were independently evaluated and double checked from available studies based on the inclusion and exclusion criteria by each of the six investigators. The following items were extracted: first author, publication year, country, tumor type, sample size, clinical stage of tumor, test methods for TUBA4B expression, outcome measures, HR value, 95\% CI of HR, survival analysis, HR extraction method, and quality score. HR, as a dominant indicator of interest, was extracted from multivariable and univariate analyses. If the articles only provided survival curves without directly detailing the HR and standard error (SE), appropriate data were extracted from the survival curves using Engauge Digitizer 4.1 as described previously [14].

For the GEO database that included TUBA4B expression and related survival data, patient data, such as OS, DFS, RFS, survival outcome, follow-up, cutoff value, HR value, and 95\% CI of HR, were extracted.

\section{Quality assessment of the primary studies}

The 2 eligible studies in our meta-analysis were each assessed for quality according to the NewcastleOttawa Scale $[15,16]$. The score of all included studies was 7 and 8 , with a mean of 7.5. A study with a higher score denoted a better methodological quality. The final scores are shown in Table 1.

\section{Fluorescence in situ hybridization}

Fluorescence in situ hybridization (FISH) kit was purchased from Shanghai Gefan Biotech Co., Ltd. (Shanghai, China) and lung cancer tissue arrays (HLugA180Su05, containing 94 patients-86 normal tissues and 94 cancer tissues) were purchased from Shanghai Outdo Biotech Co., Ltd. (Shanghai, China). Tissue sections were deparaffinized and rehydrated. FISH assay was performed according to manufacturer instructions. The probe used for IncRNA-TUBA4B was Homo 5'-FAM-ugcacuggucagccagcuuccgaauccggu-3'.

\section{Statistical analysis}

We calculated the HR values and their corresponding 95\% CI, OS, DFS, RFS, and Kaplan-Meier curves using GraphPad Prism 5.0. The cut-off value of differently expressed IncRNA-TUBA4B was set to be a $\geq 1.5$ fold difference, and the false discovery ratio was $<0.05$. We then analyzed the obtained data for OS, DFS, RFS, and other factors using STATA version 12.0 software (Stata Corporation, Collage Station, Texas, USA). For OS, DFS, and RFS, we merged the HR values and performed heterogeneity tests. $I^{2}>50 \%$ was defined as significant heterogeneity [17]. If significant heterogeneity existed between studies, we used a random effects model and performed subgroup analyses or used the fixed effects model to analyze combined HR values and $95 \% \mathrm{CI}$. All graphical representations were generated as forest plots. HR is the ratio of the prognosis in the case of high TUBA4B expression to the prognosis in the case of low TUBA4B expression. HR>1 indicates that the patients with high TUBA4B expression have a good prognosis, and $\mathrm{HR}<1$ indicates that the patients with low TUBA4B expression have a poor prognosis. For the studies from which we could obtain OS and DFS data, we constructed a funnel plot to describe publication bias using SelnHR as the abscissa and lnHR as the ordinate. We subsequently tested the funnel plot and evaluated publication bias via a linear regression model using STATA 12.0 (Begg's test and Egger's test). Sensitivity analyses were conducted for the HR and OS values of TUBA4B extracted from the individual studies using STATA version 12.0. The results of 
FISH assay were performed with the statistical program GraphPad Prism 7.0 (GraphPad, San Diego, CA, USA). Statistical analyses were performed using two-tailed Student's t-test to derive the significance of the differences between two groups. $P<0.05$ was considered statistically significant.

\section{Results}

\section{Study eligibility}

A total of 8 articles were identified from a search of the databases. After excluding 5 duplicate publications, 3 were included for further screening. After carefully reviewing the title and abstract, as well as the full text, 2 studies were finally selected based on the inclusion and exclusion criteria described in the methodology section. In addition, as shown in Fig. 1, 33 studies based on Affymetrix Human Genome U133 Plus 2.0 Array were downloaded from the GEO database.

\section{Study characteristics}

As shown in Table 1, the publication year of the articles ranged from 2016 to 2017 . A total of 230 cases from 2 included eligible studies with relevant clinical data were included in our meta-analysis. The studies included 2 types of cancers, namely lung and ovarian cancers. TUBA4B expression in these studies was all measured via quantitative real-time polymerase chain reaction.

Our meta-analysis included 3109 cases with OS (23 studies), 1568 cases with DFS (10 studies), and 887 cases with RFS (9 studies). The regions represented in the studies were USA (13), the West (14), and Asia (6). Nine types of cancer were included in the meta-analysis, namely lung $(\mathrm{n}=8)$, colon $(n=7)$, breast $(n=7)$, ovarian $(n=3)$, diffuse large B-cell lymphoma (DLBCL, $\mathrm{n}=3$ ), chronic lymphocytic leukemia (CLL, $\mathrm{n}=1$ ), glioblastoma (GBM, $n=1)$, meningioma $(n=$ $1)$, and melanoma $(n=1)$. The characteristics of these studies are summarized in Tables 2 and 3.

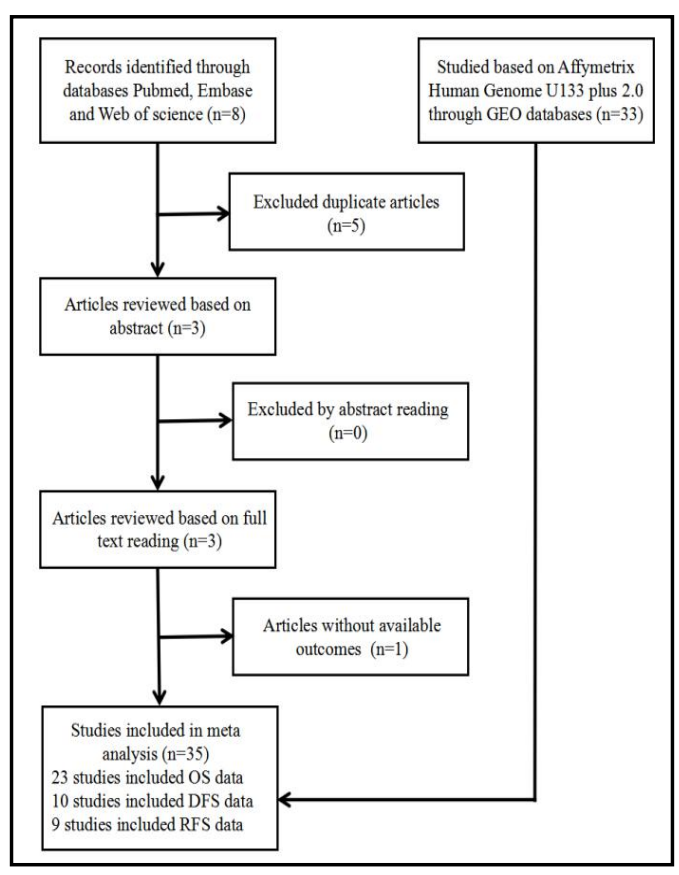

Fig. 1. Flow chart for the selection of eligible studies for meta-analysis.

Table 2. OS characteristics of studies based on Affymetrix Human Genome U133 Plus 2.0

\begin{tabular}{|c|c|c|c|c|c|c|c|c|c|}
\hline Type of cancer & GEO number & Region & No. of patients & Outcome measure & Follow up (mouth) & Cutoff value & HR & $95 \% \mathrm{CI}$ & Clinicopathological features \\
\hline Lung Cancer & GSE3141 & USA & 111 & OS & 87 & 527.700 & 1.025 & $0.843-1.516$ & None \\
\hline DLBCL & GSE10846 & USA & 414 & OS & 261 & 7.887 & 0.782 & $0.573-1.067$ & Stage \\
\hline DLBCL & GSE11318 & USA & 200 & os & 261 & 8.493 & 2.198 & $1.503-3.215$ & Stage \\
\hline Colon Cancer & GSE17536 & USA & 177 & os & 142 & 8.458 & 2.414 & $1.253-4.652$ & Stage \\
\hline Colon Cancer & GSE17538 & USA & 232 & os & 142 & 8.392 & 1.171 & $0.838-2.151$ & Grade, Stage \\
\hline CLL & GSE22762 & Germany & 107 & OS & 72 & 7.040 & 1.087 & $0.858-1.336$ & None \\
\hline Lung Cancer & GSE30219 & France & 293 & os & 256 & 6.621 & 0.858 & $0.592-1.242$ & $\mathrm{~T}, \mathrm{~N}, \mathrm{M}$ \\
\hline Lung Cancer & GSE31210 & Japan & 226 & os & 128 & 140.874 & 1.585 & $1.121-2.593$ & Stage \\
\hline Lung Cancer & GSE37745 & Sweden & 196 & os & 187 & 6.683 & 1.179 & $0.845-1.667$ & Stage \\
\hline Lung Cancer & GSE50081 & Canada & 181 & OS & 144 & 6.033 & 1.061 & $0.707-1.702$ & $\mathrm{~T}, \mathrm{~N}, \mathrm{M}$ \\
\hline Breast cancer & GSE58812 & France & 107 & os & 169 & 100.637 & 1.638 & $1.143-2.361$ & None \\
\hline GBM & GSE7696 & Switzerland & 80 & os & 72 & 7.396 & 0.966 & $0.591-1.580$ & None \\
\hline Meningioma & GSE16581 & USA & 67 & OS & 111 & 66.555 & 0.819 & $0.482-1.246$ & Grade \\
\hline Melanoma & GSE19234 & USA & 44 & os & 186 & 124.355 & 1.771 & $0.735-4.263$ & None \\
\hline Ovarian cancer & GSE19829 & USA & 28 & OS & 115 & 7.399 & 1.328 & $0.892-2.155$ & None \\
\hline Breast cancer & GSE20711 & Canada & 88 & os & 14 & 4.961 & 2.081 & $1.372-4.017$ & None \\
\hline DLBCL & GSE23501 & USA & 69 & OS & 72 & 7.622 & 1.659 & $0.955-2.261$ & None \\
\hline Lung Cancer & GSE29013 & USA & 55 & os & 82 & 6.404 & 1.448 & $1.143-1.963$ & Stage \\
\hline Colon Cancer & GSE29623 & USA & 65 & os & 120 & 8.586 & 2.031 & $1.103-6.845$ & Grade, Stage \\
\hline Ovarian Cancer & GSE30161 & USA & 58 & os & 127 & 3.615 & 2.520 & $1.370-5.003$ & Grade, Stage \\
\hline Breast cancer & GSE48390 & Taiwan & 81 & OS & 69 & 7.000 & 1.127 & $0.836-1.880$ & None \\
\hline
\end{tabular}


Zhang et al.: TUBA4B as a Prognostic Biomarker in Various Cancers

Table 3. DFS and RFS characteristics of studies based on Affymetrix Human Genome U133 Plus 2.0

\begin{tabular}{lccccccccc}
\hline Type of cancer & GEO number & Region & No. of patients & Outcome measure & Follow up (mouth) & Cutoff value & HR & $95 \%$ CI & Clinicopathological features \\
\hline Colon Cancer & GSE14333 & Australia & 226 & DFS & 142 & 6.858 & 1.330 & $0.986-1.794$ & None \\
Colon Cancer & GSE17536 & USA & 145 & DFS & 142 & 8.472 & 2.414 & $1.253-4.652$ & Stage \\
Colon Cancer & GSE17538 & USA & 200 & DFS & 142 & 8.392 & 1.171 & $0.838-2.151$ & Grade, Stage \\
Breast cancer & GSE21653 & France & 252 & DFS & 189 & 5.560 & 1.127 & $0.951-1.598$ & T, N \\
Lung Cancer & GSE30219 & France & 278 & DFS & 256 & 6.621 & 0.858 & $0.592-1.242$ & T, N, M \\
Colon Cancer & GSE38832 & USA & 92 & DFS & 111 & 7.405 & 2.416 & $0.983-10.02$ & Stage \\
Lung Cancer & GSE50081 & Canada & 177 & DFS & 144 & 6.033 & 1.061 & $0.707-1.702$ & T, N, M \\
Breast cancer & GSE6532 & Canada & 87 & DFS & 202 & 4.649 & 1.178 & $0.987-2.364$ & Grade \\
Colon Cancer & GSE29623 & USA & 53 & DFS & 120 & 8.833 & 2.031 & $1.103-6.845$ & Grade, Stage \\
Breast cancer & GSE61304 & Singapore & 58 & DFS & 85 & 4.712 & 1.552 & $1.138-2.482$ & None \\
Lung Cancer & GSE8894 & Korea & 138 & RFS & 138 & 4.191 & 1.489 & $1.192-1.967$ & None \\
Lung Cancer & GSE31210 & Japan & 226 & RFS & 128 & 140.874 & 1.585 & $1.121-2.593$ & Stage \\
Colon Cancer & GSE33114 & Netherlands & 89 & RFS & 118 & 31.900 & 1.463 & $1.106-2.293$ & None \\
Lung Cancer & GSE37745 & Sweden & 96 & RFS & 178 & 6.686 & 1.179 & $0.845-1.667$ & Stage \\
Breast cancer & GS65532 & Canada & 87 & RFS & 202 & 4.649 & 1.178 & $0.987-2.364$ & Grade \\
Breast cancer & GS5195 & Canada & 77 & RFS & 135 & 0.000 & 1.485 & $1.078-1.973$ & None \\
Breast cancer & GSE20711 & Canada & 88 & RFS & 14 & 4.961 & 2.081 & $1.372-4.017$ & None \\
Colon Cancer & GSE31595 & Denmark & 37 & RFS & 109 & 7.749 & 1.278 & $0.872-1.606$ & Stage \\
Liver cancer & GSE40873 & Japan & 49 & RFS & 73 & 6.268 & 1.720 & $1.218-4.484$ & None \\
\hline
\end{tabular}

\section{Association between TUBA4B and cancer survival \\ In total, twenty-three} studies were included for the analysis of the association of TUBA4B expression with patient survival. Random-effects model was applied to estimate the pooled HR and the $95 \%$ CI. TUBA4B was found to be significantly associated with OS in cancer patients (pooled HR $=1.33,95 \%$ CI: $1.16-1.52$, $P=0.000$; Fig. 2), and a significant heterogeneity existed between studies $\left(\mathrm{I}^{2}=59.1 \%, P=0.000\right)$. No significant publication bias (Begg's test: $\operatorname{Pr}>|\mathrm{z}|=0.162$, Egg's test: $P>|t|=0.080>$

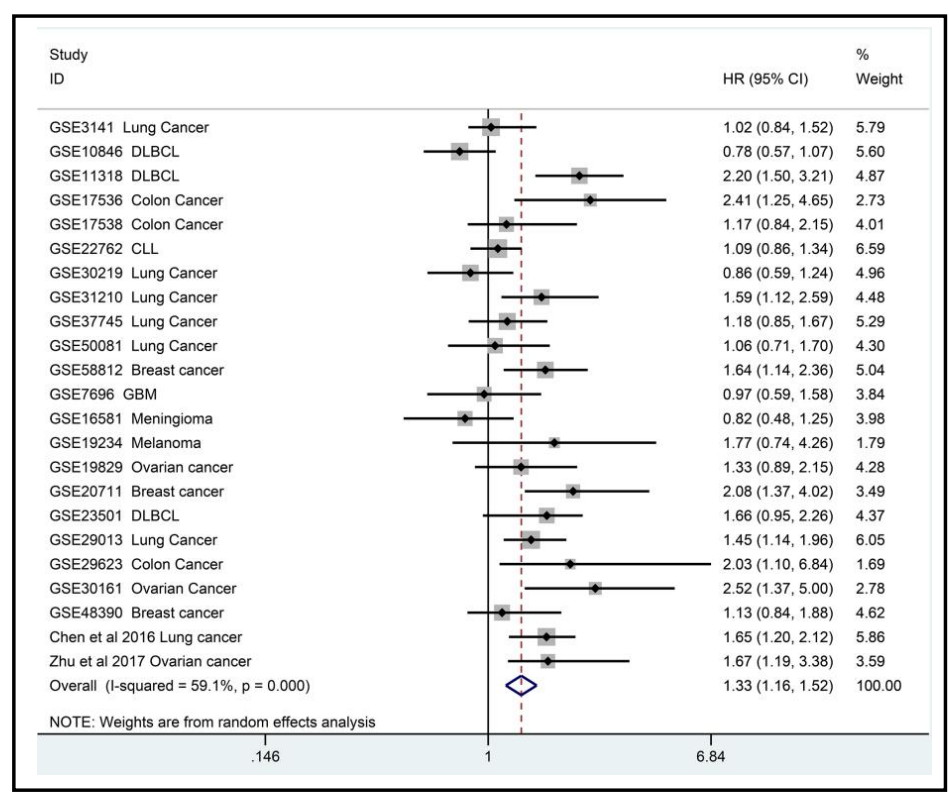

Fig. 2. Forest plot of the association between TUBA4B expression and OS in cancer patients.

0.05 ) was noted in the meta-analysis (Fig. 3A). Results of the analysis using both fixed and random effect models did not differ.

Because of heterogeneity, subgroups were analyzed for data source, region, and sample size. As shown in Fig. 4, subgroup meta-analysis of data source (GEO database vs. published articles) proved that TUBA4B was significantly associated with the OS of cancer patients in both GEO database (pooled HR $=1.30,95 \%$ CI: 1.13-1.50, $P=0.000$ ) and published articles (pooled HR $=1.66,95 \% \mathrm{CI}: 1.29-2.13, P=0.000$ ). We found a significant association between TUBA4B and the OS of cancer patients in USA (pooled HR $=1.40,95 \%$ CI: 1.11-1.76, $P=0.004$ ) and Asian countries (pooled HR $=1.51,95 \% \mathrm{CI}: 1.25-1.83, P=0.000$ ). However, the association was not significant in Western regions (pooled HR $=1.18,95 \%$ CI: 0.97-1.44, $P=0.092 ; I^{2}=48.3 \%, P=0.072$; Fig. 5). Subgroup meta-analysis of the sample size indicated a significant correlation between TUBA4B and the OS of cancer patients in both sample size of $>100$ (pooled HR $=1.29,95 \%$ CI: $1.08-1.54, P=0.005 ; \mathrm{I}^{2}=67.8 \%, P=0.000$ ) and $<100$ (pooled HR $=1.40,95 \%$ CI: 1.15-1.71, $P=0.001 ; \mathrm{I}^{2}=40.1 \%, P=0.090 ;$ Fig. 6). There was less significant heterogeneity across studies in the USA subgroup $\left(\mathrm{I}^{2}=68.2 \%, P=0.000\right)$ as well as studies from the GEO database $\left(\mathrm{I}^{2}=59.4 \%, P=0.000\right)$. No heterogeneity existed in the Asian subgroup $\left(I^{2}=0.0 \%, P=0.459\right)$ and in the data collected from published articles $\left(I^{2}=0.0 \%\right.$, $P=0.967)$. 

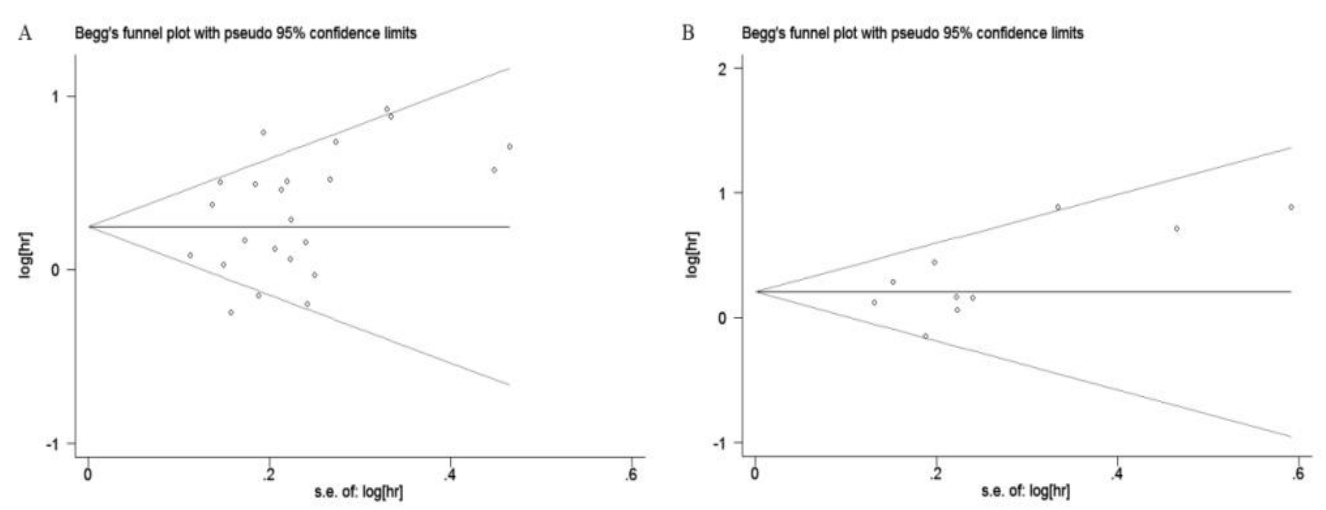

C Begg's funnel plot with pseudo 95\% confidence limits

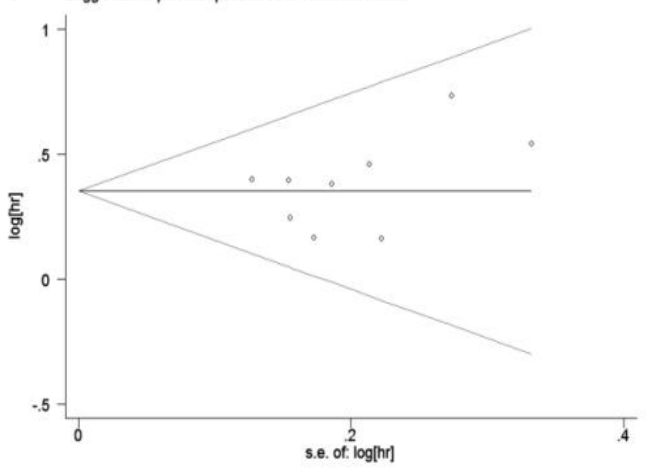

Fig. 3. Funnel plot analysis of potential publication bias for TUBA4B expression with OS, DFS, and RFS in cancer patients. A. No significant publication bias existed for OS (Begg's test: $\operatorname{Pr}>|z|=0.162$, Egg's test: $P>$ $|t|=0.080>0.05$ ). B. No significant publication bias existed for DFS (Begg's test: $\operatorname{Pr}>|z|=0.128$, Egg's test: $P>|t|=0.087>0.05$ ). C. No significant publication biases existed for RFS (Begg's test: $\operatorname{Pr}>|z|=0.404$, Egg's test: $P>|t|=0.355>0.05)$.

Fig. 4. Forest plot of the subgroup meta-analysis of the association between TUBA4B expression and OS in cancer patients based on published articles and the GEO database.

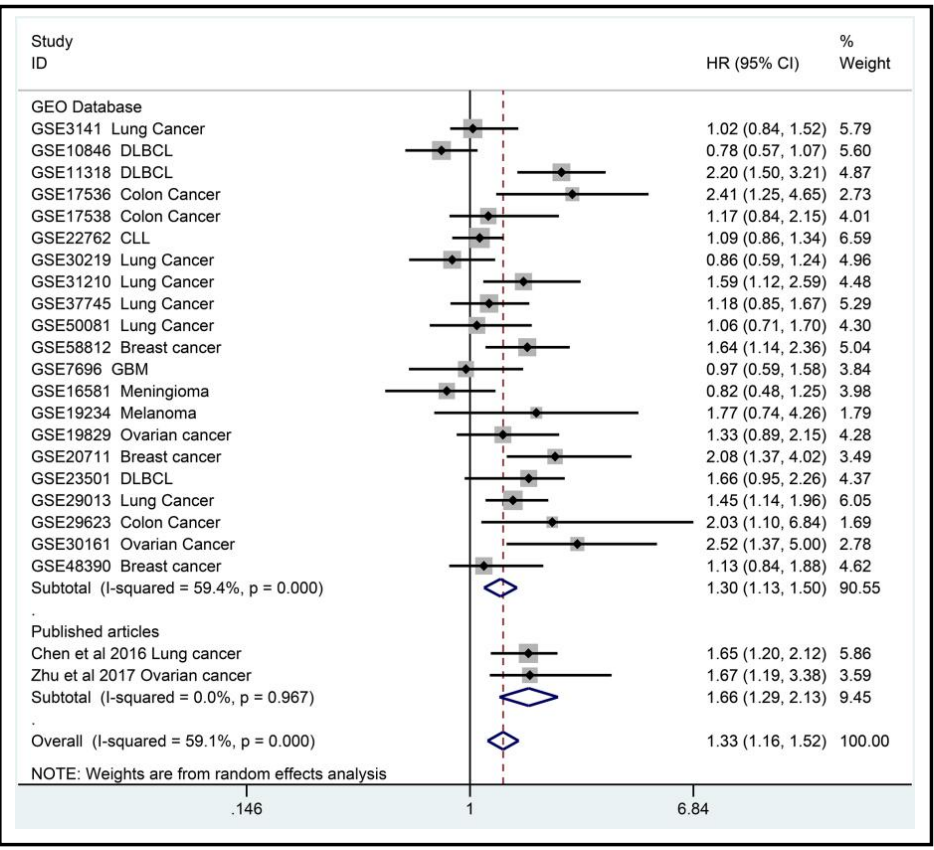


Fig. 5. Forest plot of the subgroup meta-analysis of the association between TUBA4B expression and OS in cancer patients based on regions.

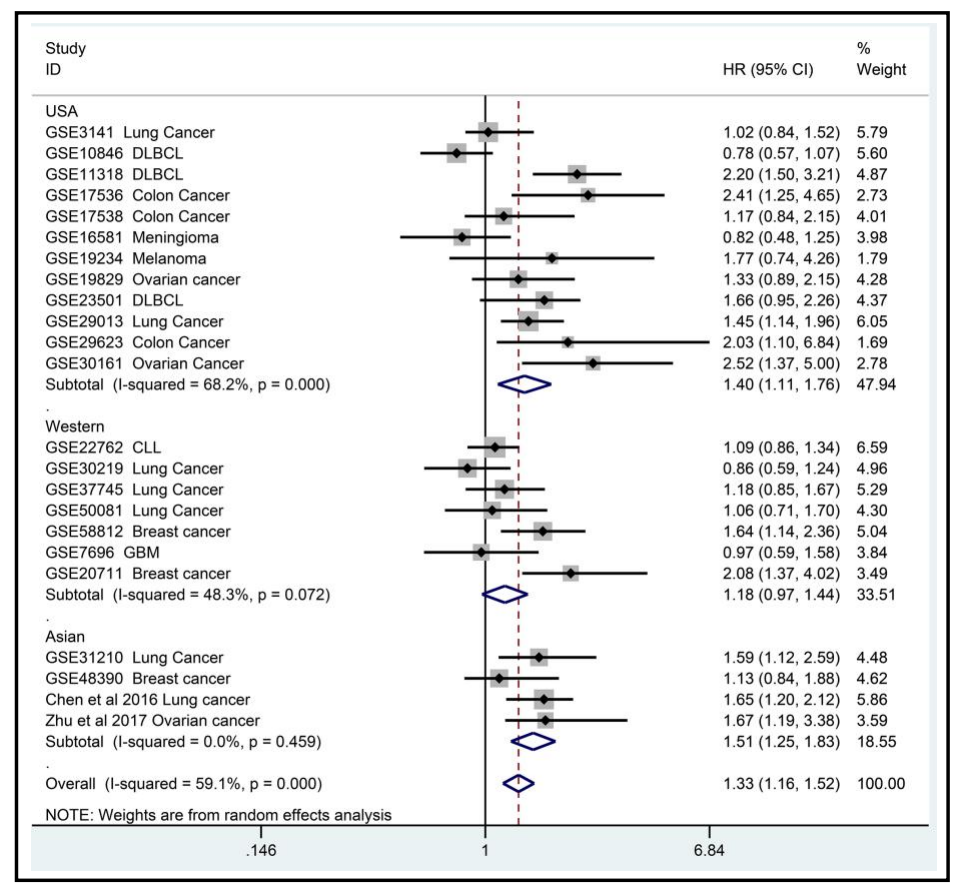

Fig. 6. Forest plot of the subgroup meta-analysis of the association between TUBA4B expression and OS in cancer patients based on sample size.

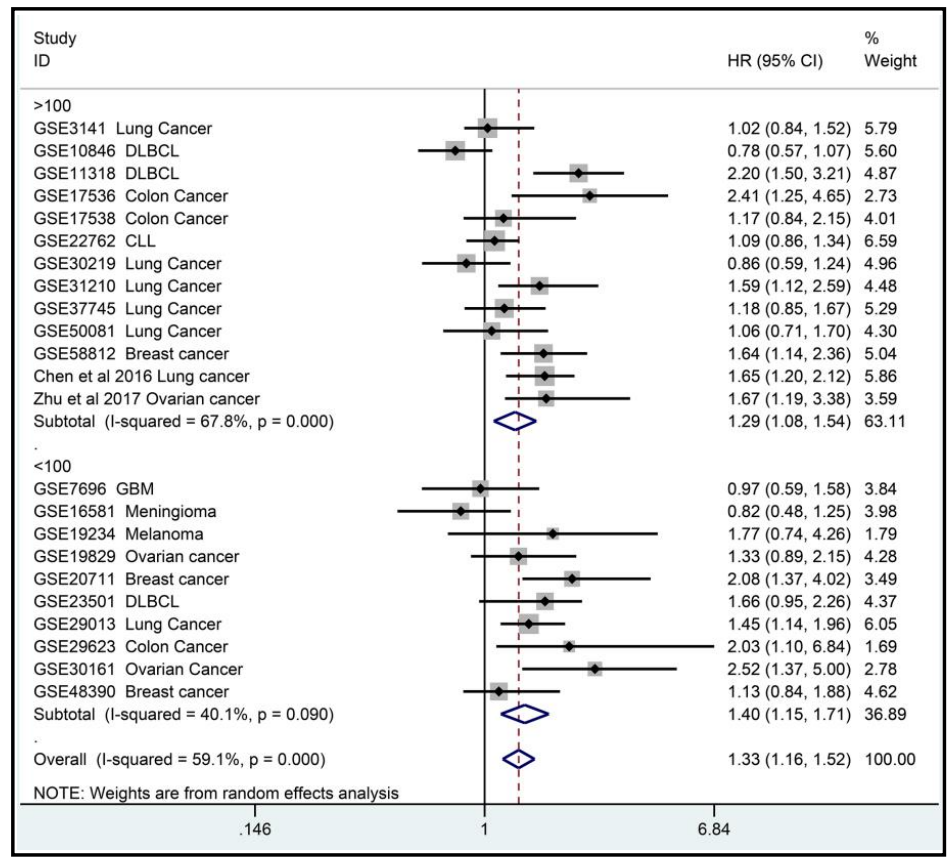

To maximize clinical relevance, subgroups were analyzed based on tumor types. We found that TUBA4B was a risk factor for the poor prognosis of lung cancer (pooled $\mathrm{HR}=1.24$, 95\% CI: 1.03-1.49, $P=0.021$ ), colon cancer (pooled HR $=1.67,95 \% \mathrm{CI}: 1.02-2.74, P=0.042$ ), breast cancer (pooled HR =1.52, 95\% CI: 1.10-2.12, $P=0.012$ ), and ovarian cancer (pooled HR $=1.67,95 \%$ CI: 1.18-2.36, $P=0.004)$. However, no significant association was noted between TUBA4B and OS for DLBCL, CLL, and neurotumors. These results were strengthened by the low heterogeneity between the studies (Fig. 7). Subgroup analysis of pooled HR of OS in different types of cancer with decreased TUBA4B expression, 95\% CI, heterogeneity, and overall effect are detailed in Table 4. Since lung cancer had six GEO datasets and one article (Fig. 7) and had the largest number of patients in the classification of tumor types (Table 4), 


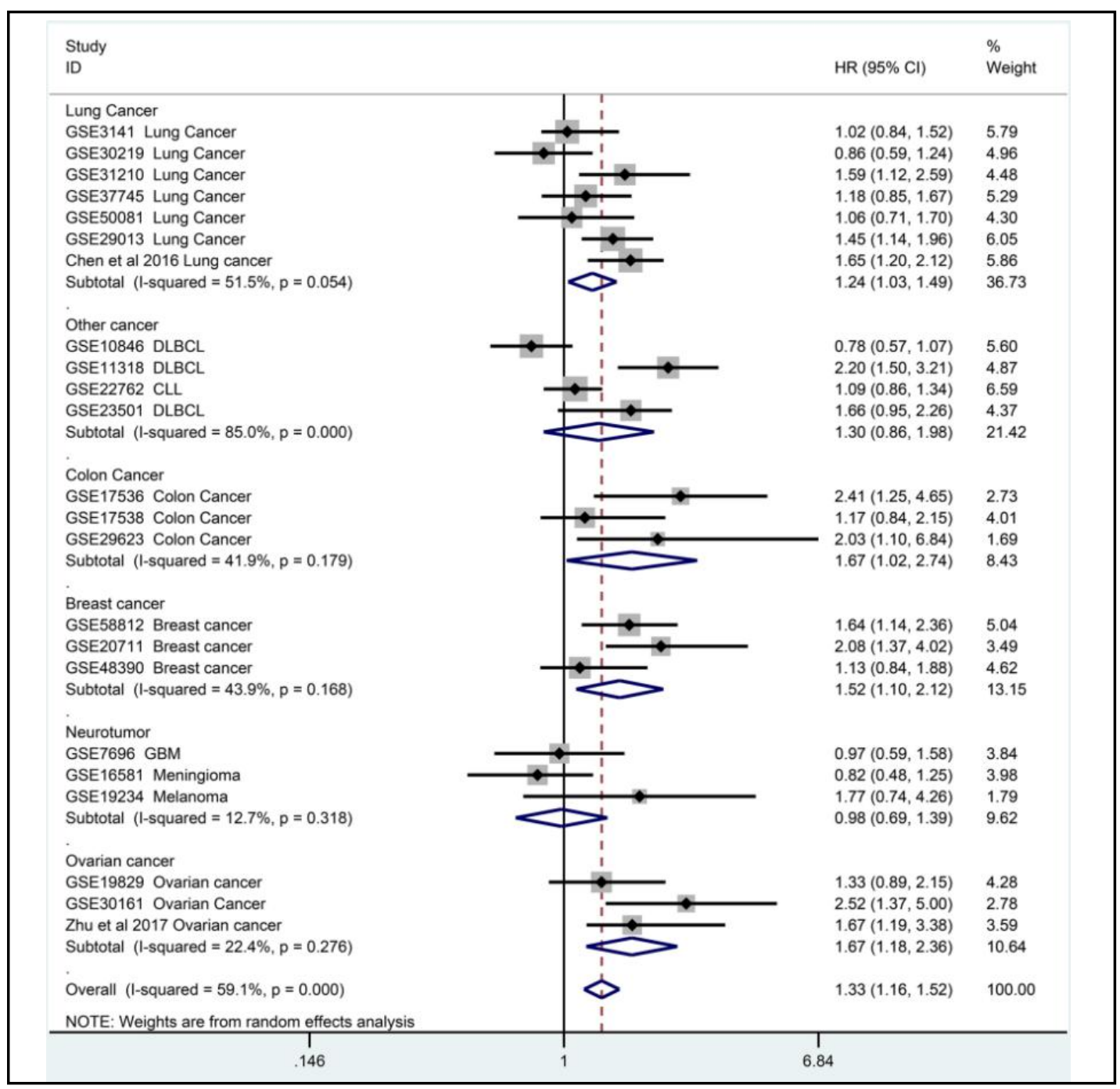

Fig. 7. Forest plot of the subgroup meta-analysis of the association between TUBA4B expression and OS in cancer patients based on tumor types.

Table 4. Results of the subgroup analysis of pooled hazard ratios of OS in different types of cancer with decreased TUBA4B expression

\begin{tabular}{|c|c|c|c|c|c|c|c|c|}
\hline \multirow[t]{2}{*}{ Subgroup analysis } & \multirow[t]{2}{*}{ No. of studies } & \multirow[t]{2}{*}{ No. of patients } & \multicolumn{2}{|c|}{ Pooled HR $(95 \% \mathrm{CI})$} & \multicolumn{2}{|c|}{ Heterogeneity } & \multicolumn{2}{|c|}{ Overall effect } \\
\hline & & & Fix & Random & $\mathrm{I}^{2}$ & $\mathrm{P}$ & $\mathrm{Z}$ & $\mathrm{P}$ \\
\hline OS & 23 & 3109 & $1.28[1.18,1.39]$ & $1.33[1.16,1.52]$ & $59.1 \%$ & 0.000 & 4.19 & 0.000 \\
\hline \multicolumn{9}{|l|}{ Data source } \\
\hline Published articles & 2 & 230 & $1.66[1.29,2.13]$ & $1.66[1.29,2.13]$ & $0.0 \%$ & 0.967 & 3.94 & 0.000 \\
\hline GEO & 21 & 2879 & $1.24[1.14,1.35]$ & $1.30[1.13,1.50]$ & $59.4 \%$ & 0.000 & 3.64 & 0.000 \\
\hline \multicolumn{9}{|l|}{ Region } \\
\hline USA & 12 & 1520 & $1.30[1.15,1.46]$ & $1.40[1.11,1.76]$ & $68.2 \%$ & 0.000 & 2.88 & 0.004 \\
\hline Western & 7 & 1052 & $1.16[1.02,1.33]$ & $1.18[0.97,1.44]$ & $48.3 \%$ & 0.072 & 1.68 & 0.092 \\
\hline Asian & 4 & 537 & $1.51[1.25,1.83]$ & $1.51[1.25,1.83]$ & $0.0 \%$ & 0.459 & 4.23 & 0.000 \\
\hline \multicolumn{9}{|l|}{ Tumor type } \\
\hline Lung cancer & 7 & 1176 & $1.26[1.11,1.42]$ & $1.24[1.03,1.49]$ & $51.5 \%$ & 0.054 & 2.30 & 0.021 \\
\hline Other cancer & 4 & 790 & $1.19[1.02,1.38]$ & $1.30[0.86,1.98]$ & $85.0 \%$ & 0.000 & 1.24 & 0.216 \\
\hline Colon cancer & 3 & 474 & $1.57[1.10,2.23]$ & $1.67[1.02,2.74]$ & $41.9 \%$ & 0.179 & 2.04 & 0.042 \\
\hline Breast cancer & 3 & 276 & $1.50[1.18,1.92]$ & $1.52[1.10,2.12]$ & $43.9 \%$ & 0.168 & 2.51 & 0.012 \\
\hline Neurotumor & 3 & 191 & $0.97[0.71,1.33]$ & $0.98[0.69,1.39]$ & $12.7 \%$ & 0.318 & 0.10 & 0.918 \\
\hline Ovarian cancer & 3 & 202 & $1.64[1.22,2.21]$ & $1.67[1.18,2.36]$ & $22.4 \%$ & 0.276 & 2.92 & 0.004 \\
\hline \multicolumn{9}{|l|}{ Sample size } \\
\hline$>100$ & 13 & 2474 & $1.24[1.12,1.36]$ & $1.29[1.08,1.54]$ & $67.8 \%$ & 0.000 & 2.81 & 0.005 \\
\hline$<100$ & 10 & 635 & $1.38[1.19,1.60]$ & $1.40[1.15,1.71]$ & $40.1 \%$ & 0.090 & 3.29 & 0.001 \\
\hline
\end{tabular}




\section{Cellular Physiology Cell Physiol Biochem 2018;49:530-544}

\begin{tabular}{ll|l} 
DOI: 10.1159/000492991 & O 2018 The Author(s). Published by S. Karger AG, Basel \\
and Biochemistry & Published online:29 August, 2018 & wwwwkargercom/cpb
\end{tabular}

Fig. 8. Fluorescence in situ hybridization (FISH) of lung cancer tissues. A, C. Representative images and FISH scores analysis of lncRNA-TUBA4B staining in normal and tumor lung tissues (scale bar, $100 \mu \mathrm{m}$ ). B, D. Representative images and FISH scores analysis of IncRNATUBA4B staining at stages I-IV (scale bar, $100 \mu \mathrm{m}$ ); two-tailed Student's t-test, ${ }^{*} \mathrm{P}<0.05, \quad{ }^{* *} \mathrm{P}<0.01$ and $* * * \mathrm{P}<0.001$.
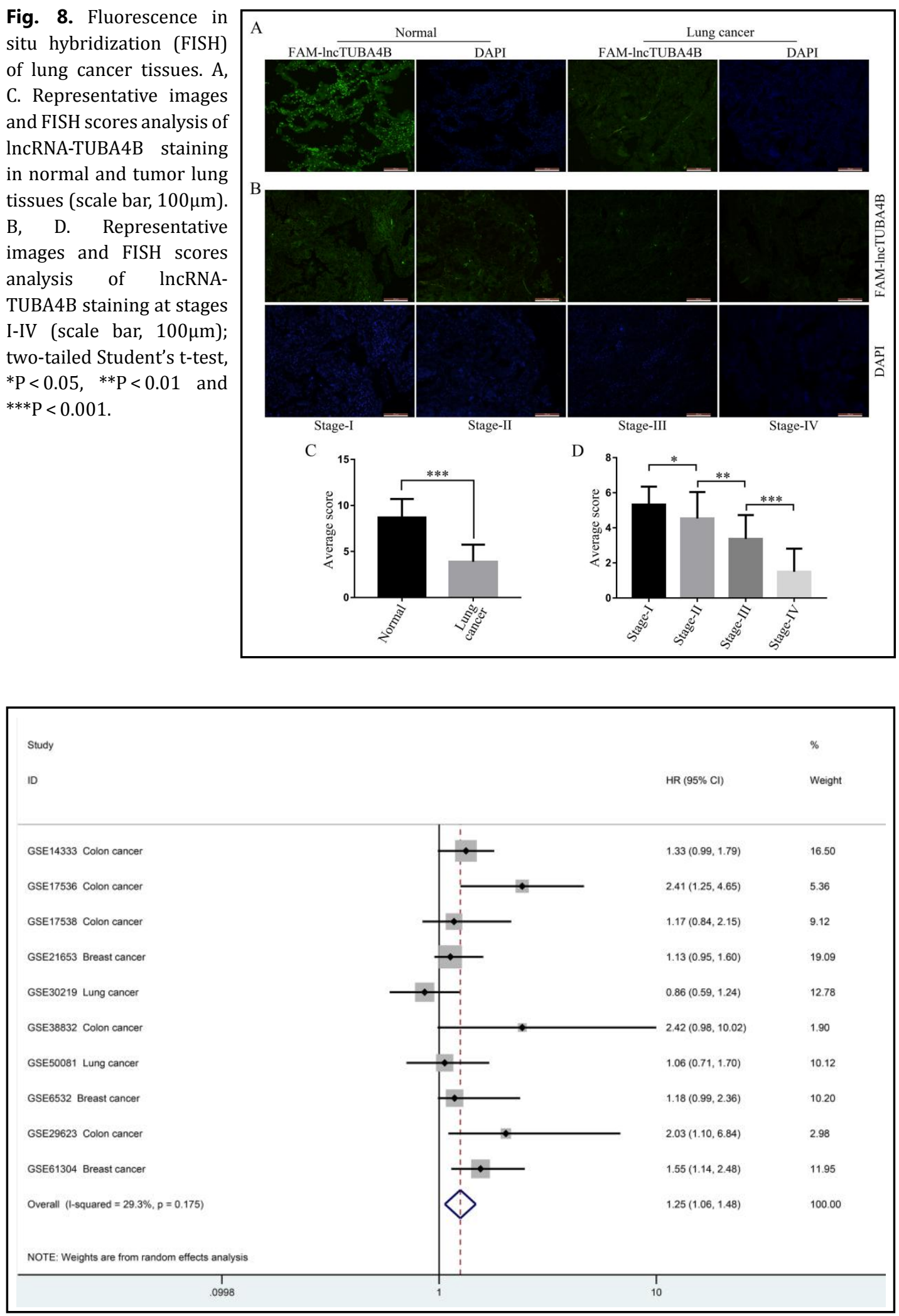

Fig. 9. Forest plot of the association between TUBA4B expression and DFS in cancer patients. 
we evaluated the expression of TUBA4B in lung cancer tissues for experimental verification by FISH assay. It was found that the lncRNA-TUBA4B was significantly lower expression in tumor tissues than normal lung tissues (Fig. 8A, C). In addition, we found that the expression of IncRNA-TUBA4B was decreased with the progression of lung cancer stage (Fig. 8B, D). These results indicate that the patients with low TUBA4B expression have a poor prognosis.

Study
ID
GSE17536 Colon cancer
GSE17538 Colon cancer
GSE21653 Breast cancer
GSE30219 Lung cancer
GSE38832 Colon cancer
GSE50081 Lung cancer
GSE6532 Breast cancer
GSE29623 Colon cancer
GSE61304 Breast cancer
Overall (1-squared = 29.3\%, $\mathrm{p}=0.175)$

Fig. 10. Forest plot of the subgroup meta-analysis of the association between TUBA4B expression and DFS in cancer patients based on cancer types.

Table 5. Results of subgroup analysis of pooled hazard ratios of DFS and RFS in different types of cancer with decreased TUBA4B expression

\begin{tabular}{|c|c|c|c|c|c|c|c|c|}
\hline \multirow[t]{2}{*}{ Subgroup analysis } & \multirow[t]{2}{*}{ No. of studies } & \multirow[t]{2}{*}{ No. of patients } & \multicolumn{2}{|c|}{ Pooled HR $(95 \% \mathrm{CI})$} & \multicolumn{2}{|c|}{ Heterogeneity } & \multicolumn{2}{|c|}{ Overall effect } \\
\hline & & & Fix & Random & $\mathrm{I}^{2}$ & $\mathrm{P}$ & $\mathrm{Z}$ & $P$ \\
\hline DFS & 10 & 1568 & $1.23[1.08,1.40]$ & $1.25[1.06,1.48]$ & $29.3 \%$ & 0.175 & 2.68 & 0.007 \\
\hline \multicolumn{9}{|l|}{ Tumor type } \\
\hline Colon cancer & 5 & 716 & $1.45[1.16,1.82]$ & $1.49[1.15,1.95]$ & $14.8 \%$ & 0.320 & 2.98 & 0.003 \\
\hline Breast cancer & 3 & 397 & $1.23[1.01,1.49]$ & $1.23[1.01,1.49]$ & $0.0 \%$ & 0.398 & 2.10 & 0.036 \\
\hline Lung cancer & 2 & 455 & $0.94[0.71,1.24]$ & $0.94[0.71,1.24]$ & $0.0 \%$ & 0.469 & 0.45 & 0.653 \\
\hline RFS & 9 & 887 & $1.42[1.26,1.60]$ & $1.42[1.26,1.60]$ & $0.0 \%$ & 0.747 & 5.81 & 0.000 \\
\hline \multicolumn{9}{|l|}{ Tumor type } \\
\hline Lung cancer & 3 & 460 & $1.41[1.17,1.69]$ & $1.41[1.18,1.69]$ & $0.0 \%$ & 0.461 & 3.70 & 0.000 \\
\hline Other cancer & 3 & 175 & $1.39[1.11,1.73]$ & $1.39[1.11,1.73]$ & $0.0 \%$ & 0.678 & 2.92 & 0.003 \\
\hline Breast cancer & 3 & 252 & $1.48[1.18,1.86]$ & $1.49[1.14,1.94]$ & $23.0 \%$ & 0.273 & 2.93 & 0.003 \\
\hline \multicolumn{9}{|l|}{ Region } \\
\hline Asian & 3 & 413 & $1.53[1.25,1.88]$ & $1.53[1.25,1.88]$ & $0.0 \%$ & 0.907 & 4.10 & 0.000 \\
\hline Western & 6 & 474 & $1.37[1.18,1.59]$ & $1.37[1.18,1.59]$ & $0.0 \%$ & 0.530 & 4.21 & 0.000 \\
\hline \multicolumn{9}{|l|}{ Sample size } \\
\hline$>100$ & 2 & 364 & $1.51[1.22,1.88]$ & $1.51[1.22,1.88]$ & $0.0 \%$ & 0.802 & 3.78 & 0.000 \\
\hline$<100$ & 7 & 523 & $1.38[1.20,1.60]$ & $1.38[1.20,1.60]$ & $0.0 \%$ & 0.599 & 4.46 & 0.000 \\
\hline
\end{tabular}




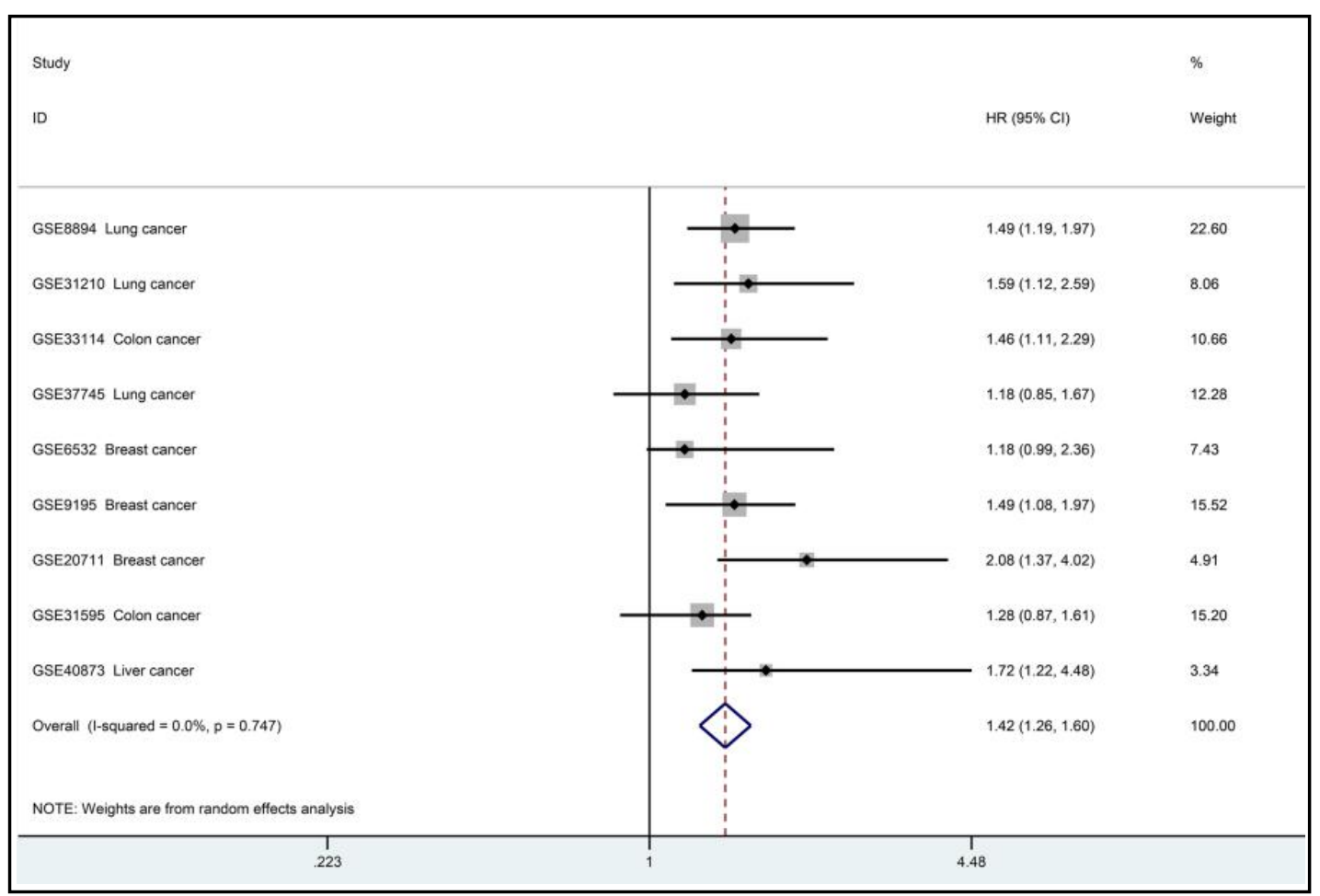

Fig. 11. Forest plot of the association between TUBA4B expression and RFS in cancer patients.

Meta-analysis of the association between TUBA4B expression and DFS

A total of 10 studies were included in the analysis of the association between TUBA4B expression and DFS in cancer patients. In the meta-analysis, fixed and random effect models were used to estimate the pooled HR and respective 95\% CI. The results showed that TUBA4B was significantly associated with DFS (pooled HR = 1.25, 95\% CI: 1.06-1.48, $P=0.007$; Fig. 9). Neither significant heterogeneity $\left(\mathrm{I}^{2}=29.3 \%, P=0.175\right)$ nor significant publication bias (Begg's test: $\operatorname{Pr}>|z|=0.128$, Egg's test: $\mathrm{P}>|\mathrm{t}|=0.087>0.05$ ) existed across the studies (Fig. 3B). A subgroup analysis found that TUBA4B was significantly associated with DFS in colon cancer (pooled HR = 1.49, 95\% CI: 1.15-1.95; $\mathrm{I}^{2}=14.8 \%, P=0.320$ ) and breast cancer (pooled HR = 1.23, 95\% CI: 1.01-1.49; $\mathrm{I}^{2}=0.0 \%, P=0.398$ ) (Fig. 10, Table 5).

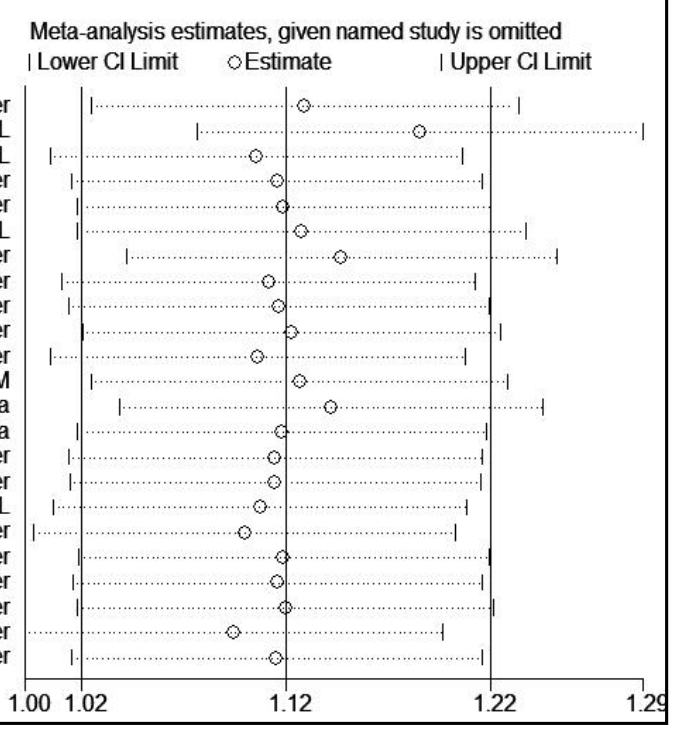

Fig. 12. Sensitivity analysis of the effect of the individual study on the pooled HR of OS. 


\section{Cellular Physiology Cell Physiol Biochem 2018:49:530-544 and Biochemistry Publ: 10.1159/000492991 2018 The Author(s). Published by S. Karger AG, Basel

Meta-analysis of the association between TUBA4B expression and RFS

A total of 9 studies were included in the meta-analysis of TUBA4B expression and RFS. The HR of the association between decreased TUBA4B expression and RFS in these cancer patients was $1.42(95 \%$ CI: $1.26-1.60, P=0.000$; Fig. 11). There was no significant heterogeneity $\left(\mathrm{I}^{2}=0.0 \%, \quad P=0.1747\right)$ and publication bias (Begg's test: $\operatorname{Pr}>|\mathrm{z}|=$ 0.404, Egg's test: $P>|t|$ $=0.355>0.05$ ) across the studies (Fig. 3C). We used both fixed and random effect models to calculate the effect of TUBA4B on patient survival and found that the results were not markedly different.

\section{Sensitivity analysis}

Sensitivity analysis was performed by removing each study alternately from the pooled analysis. This analysis aimed to evaluate the effect of the removed study on the pooled HRs. Removing any included study had no significant impact on the meta-analysis outcomes, suggesting the robustness of the results. The sensitivity analysis revealed that the pooled HR of OS (Fig. 12), DFS (Fig. 13), and RFS (Fig. 14) were reliable.

\section{Discussion}

This meta-analysis used the online databases PubMed, Embase, and Web of Science and the GEO database to evaluate the association between TUBA4B expression and survival according to clinical indicators. A total of 23 studies comprising 3109 samples of OS were included in this meta-analysis, in which 21 studies were identified via the GEO database, representing 2879 samples. At present, only few clinical studies have focused on TUBA4B because of the small number of patients with such condition. Therefore, a classical meta-
Fig. 13. Sensitivity analysis of the effect of the individual study on the pooled HR of DFS.

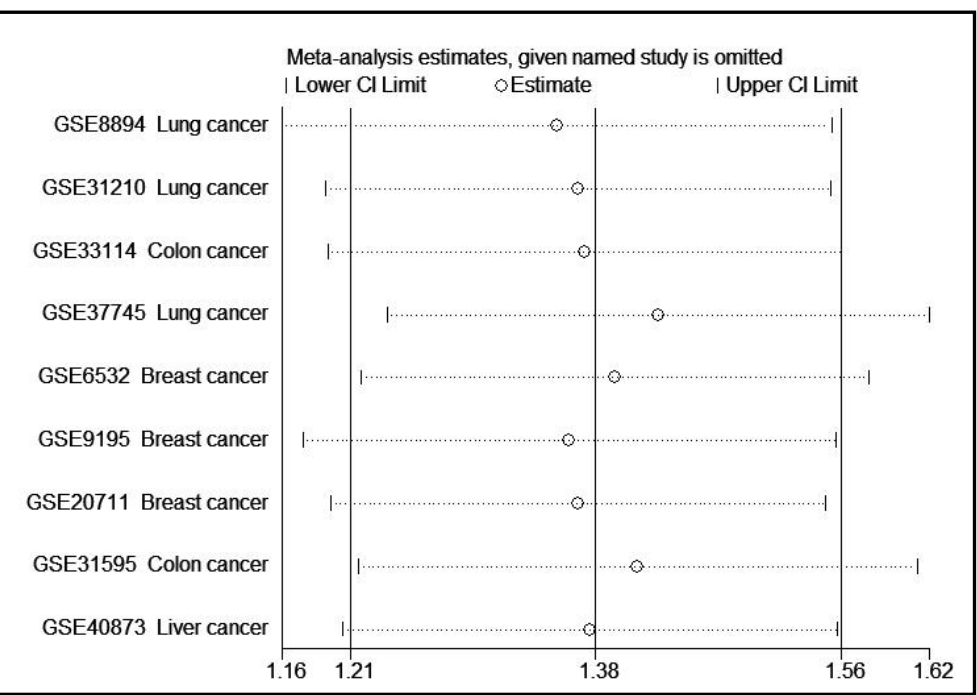

Fig. 14. Sensitivity analysis of the effect of the individual study on the pooled HR of RFS. 


\section{Cellular Physiology Cell Physiol Biochem 2018;49:530-544 \begin{tabular}{ll|l} 
and Biochemistry & $\begin{array}{l}\text { DOI: 10.1159/000492991 } \\
\text { Published online: 29 August, } 2018\end{array}$ & $\begin{array}{l}\text { O 2018 The Author(s). Published by S. Karger AG, Basel } \\
\text { www.karger.com/cpb }\end{array}$ \\
\cline { 2 - 3 }
\end{tabular} \\ Zhang et al.: TUBA4B as a Prognostic Biomarker in Various Cancers}

analysis may overestimate the effect of TUBA4B in tumors. The establishment of tumor databases, such as GEO and The Cancer Genome Atlas, allows for a more extensive and accurate study of tumor molecular markers $[18,19]$. The inclusion of GEO data allows for a more extensive data source and more realistic results.

At present, only 2 articles demonstrated that low IncRNA-TUBA4B may be a predictor of poor prognosis in non-small-cell lung cancer and ovarian cancer $[12,13]$. However, the sample size of these studies is small. This meta-analysis evaluated the value of TUBA4B as a marker of tumor prognosis. Through systematic analysis, we found that TUBA4B expression was low in many types of tumors. We evaluated the expression of TUBA4B in lung cancer tissue arrays for experimental verification by FISH assay and the results were consistent with the analysis. LncRNA-TUBA4B was lower expression in tumor tissues than normal lung tissues $(P<0.001)$. By combining the HRs from Cox analysis, we found that TUBA4B was an independent risk factor for OS in cancer patients (pooled HR $=1.33,95 \%$ CI: 1.16-1.52, $P=0.000$ ). In addition, TUBA4B can be considered an independent prognostic risk factor for DFS (HR $=1.25,95 \% \mathrm{CI}: 1.06-1.48, P=0.007)$ and RFS (HR $=1.42,95 \%$ CI: $1.26-1.60$, $P=0.000$ ) in cancer patients.

To explore the heterogeneity, subgroups were analyzed, and the results revealed that TUBA4B expression can be used as a prognostic factor for OS, DFS, and RFS. We found that TUBA4B significantly affected RFS (HR $=1.42,95 \%$ CI: $1.26-1.60, P=0.000)$, and the heterogeneity $\left(\mathrm{I}^{2}=0.0 \%, P=0.1747\right)$ was small, thereby strengthening our results. Moreover, both Begg's and Egg's tests identified no significant publication bias about the independent prognostic role of TUBA4B in OS, DFS, and RFS. A sensitivity analysis using both fixed and random effect models showed no significant differences. In summary, our analysis is reliable, and the low expression of TUBA4B is associated with poor prognosis.

In contrast, our meta-analysis has several limitations. First, differences in article quality across studies can cause bias in the meta-analysis. Second, TUBA4B expression in 2 published articles are not directly obtained from the primary studies and were estimated using a software, and this could lead to an error in the HR value and may also increase the original value of heterogeneity. Third, the length of survival and treatment of patients was significantly correlated, and these differences might markedly affect HR and thus result in heterogeneity. In addition, most included papers reported positive results, which may generate publication bias. Therefore, the significance of TUBA4B in predicting the prognosis of cancer patients may be slightly overestimated. Thus, the study results must be confirmed in a larger sample in a polycentric and randomized controlled prospective study.

\section{Conclusion}

The low expression of TUBA4B was significantly associated with poor OS, DFS, and RFS in cancer patients. The results of our meta-analysis suggest that TUBA4B can be a novel biomarker for the prognosis of various cancers. Further research is needed to explore the role of TUBA4B in human cancer.

\section{Abbreviations}

lncRNAs (long non-coding RNAs); GEO (Gene Expression Omnibus); TUBA4B (tubulin alpha 4b); OS (overall survival); DFS (disease-free survival); RFS (recurrence-free survival); HR (hazard ratio); CI (confidence interval); GBM (glioblastoma); CLL (chronic lymphocytic leukemia); DLBCL (diffuse large B-cell lymphoma). 


\section{Cellular Physiology Cell Physiol Biochem 2018;49:530-544 \begin{tabular}{l|l} 
and Biochemistry Publishe.1159/000492991 & $\begin{array}{l}\text { (c) } 2018 \text { The Author(s). Published by S. Karger AG, Basel } \\
\text { www.karger.com/cpb }\end{array}$
\end{tabular}

\section{Acknowledgements}

This study was funded by the Foundation of Sichuan Province Education Office (17TD0012), the Foundation of Sichuan Province Health and Family Planning Commission (16ZD040), the Foundation of Sichuan Province Medical Association (Q16015), the Foundation of The First Affiliated Hospital of Chengdu Medical College (CYFY2017ZD03), and the Foundation of Chengdu Medical College (CYTD15-03, CYZ14-015, 16Z139).

$\mathrm{Y} \mathrm{Xu}$, T Zhang, and DM Wu conceived and designed this study. SH Deng, R Han, T Liu, and $\mathrm{J} \mathrm{Li} \mathrm{searched} \mathrm{databases} \mathrm{and} \mathrm{extracted} \mathrm{the} \mathrm{data.} \mathrm{DM} \mathrm{Wu} \mathrm{and} \mathrm{T} \mathrm{Zhang} \mathrm{performed} \mathrm{the}$ statistical analysis. T Zhang wrote the manuscript. All authors reviewed and approved the final manuscript.

\section{Disclosure Statement}

The authors have no competing financial interests to disclose.

\section{References}

1 Torre LA, Bray F, Siegel RL, Ferlay J, Lortet-Tieulent J, Jemal A: Global cancer statistics, 2012. CA Cancer J Clin 2015;65:87-108.

- Miller KD, Siegel RL, Lin CC, Mariotto AB, Kramer JL, Rowland JH, Stein KD, Alteri R, Jemal A: Cancer treatment and survivorship statistics, 2016. CA Cancer J Clin 2016;66:271.

3 Li DX, Fei XR, Dong YF, Cheng CD, Yang Y, Deng XF, Hunag HL, Niu WX, Zhou CX, XIA CY, Niu CS: The long noncoding RNA CRNDE acts as a ceRNA and promotes glioma malignancy by preventing miR-136-5p-mediated downregulation of Bcl-2 and Wnt2. Oncotarget 2017;8:88163-88178.

-4 Huang W, Zhang X, Li A, Xie, L, Miao, X:Differential regulation of mRNAs and lncRNAs related to lipid metabolism in two pig breeds. Oncotarget 2017;8:87539-87553.

5 Ishikawa T, Nishida T, Ono M, Takarada T, Kurihara S, Furumatsu T, Murase Y, Takigawa M, Oohashi T, Kamioka H, Kubota S: Physiological role of urothelial cancer-associated 1 long noncoding RNA in human skeletogenic cell differentiation. J Cell Physiol 2017 DOI: 10.1002/jcp.26285.

6 Jiang P, Chen A, Wu X, Zhou M, Haq IU, Mariyam Z, Feng Q: NEAT1 acts as an inducer of cancer stem cell-like phenotypes in NSCLC by inhibiting EGCG-upregulated CTR1. J Cell Physiol 2017 DOI: 10.1002/jcp.26288.

7 Xie H, Liao X, Chen Z, Fang Y, He A, Zhong Y, Gao Q, Xiao H, Li J, Hunag W, Liu Y: LncRNA MALAT1 inhibits apoptosis and promotes invasion by antagonizing miR-125b in bladder cancer cells. J Cancer 2017;8:38033811.

-8 Gibb EA, Brown CJ, Lam WL: The functional role of long non-coding RNA in human carcinomas. Mol Cancer 2011;10:38.

$>9$ Da M, Ma J, Zhang Y, Yang J, Yao J, Huang B, Ma H, Ge L: High expression level of long non-coding RNA HOTAIR is associated with poor overall survival in gastric cancer patients: evidence from meta-analysis. J BUON 2017;22:911-918.

10 Xu X, Xu Y, Shi C, Wang B, Yu X, Zou Y, Hu T: A genome-wide comprehensively analyses of long noncoding RNA profiling and metastasis associated lncRNAs in renal cell carcinoma. Oncotarget 2017;8:87773-87781.

11 Zhang H, Chen Z, Wang X, Huang Z, He Z, Chen Y: Long non-coding RNA: a new player in cancer. J Hematol Oncol 2013, 6:37.

12 Chen J, Hu L, Wang J, Zhang F, Chen J, XU G, Wang Y, Pan Q: Low expression LncRNA TUBA4B is a poor predictor of prognosis and regulates cell proliferation in non-small cell lung cancer. Pathol Oncol Res 2017;23:265-270.

13 Zhu FF, Zheng FY, Wang HO, Zheng JJ, Zhang Q: Downregulation of lncRNA TUBA4B is associated with poor prognosis for epithelial ovarian cancer. Pathol Oncol Res 2017 DOI: 10.1007/s12253-017-0258-7.

14 Hu Y, Ma Z, He Y, Liu W, Su Y, Tang Z: LncRNA-SNHG1 contributes to gastric cancer cell proliferation by regulating DNMT1. Biochem Biophys Res Commun 2017;491:926-931. 


\section{Cellular Physiology Cell Physiol Biochem 2018:49:530-544 \begin{tabular}{l|l|l} 
DOI: 10.1159/000492991 & $\begin{array}{l}\text { O 2018 The Author(s). Published by S. Karger AG, Basel } \\
\text { www.karger.com/cpb }\end{array}$ \\
\hline
\end{tabular} \\ Zhang et al.: TUBA4B as a Prognostic Biomarker in Various Cancers}

15 Stang A: Critical evaluation of the Newcastle-Ottawa scale for the assessment of the quality of nonrandomized studies in meta-analyses. Eur J Epidemiol 2010;25:603-605.

16 Hu H, Cai W, Zheng S, Ge W: SPARCL1, a novel prognostic predictive factor for GI malignancies: A metaanalysis. Cell Physiol Biochem 2017;44:1485-1496.

17 Egger M, Davey SG, Schneider M, Minder C: Bias in meta-analysis detected by a simple, graphical test. BMJ 1997;315:629-634.

18 Barrett T, Wilhite S E, Ledoux P, Evangelista C, Kim IF, Tomashevsky M, Marshall KA, Phillippy KH, Sherman PM. Holko M, Yefanov A, Lee H, Zhang N, Robertson CL, Serova N, Davis S, Soboleva A: NCBI GEO: archive for functional genomics data sets-update. Nucleic Acids Res 2013;41:991-995.

-19 Cancer Genome Atlas Research Network, Mclendon R, Friedman A, Bigner D, et al: Comprehensive genomic characterization defines human glioblastoma genes and core pathways. Nature 2008;455:1061-1068. 